\title{
Regulation of Body Weight and Metabolism by Tanycyte-Derived Neurogenesis in Young Adult Mice
}

\author{
Seth Blackshaw, Daniel A. Lee, Thomas Pak, and Sooyeon Yoo
}

\begin{abstract}
The hypothalamus controls many homeostatic and instinctive physiological processes, including the sleep-wake cycle, food intake, and sexually dimorphic behaviors. These behaviors are regulated by environmental and physiological cues, although the molecular and cellular mechanisms that underlie these effects are still poorly understood. Recently, it has become clear that both the juvenile and adult hypothalamus exhibit neurogenesis, which modifies homeostatic neural circuitry. In this manuscript, we report data addressing the role of sex-specific and dietary factors in controlling neurogenesis in the mediobasal hypothalamus. We report that a high fat diet (HFD) activates neurogenesis in the median eminence (ME) of young adult female, but not male mice, and that focal irradiation of the ME in HFD-fed mice reduces weight gain in females, but not males. These results suggest that some physiological effects of HFD are mediated by sexually dimorphic neurogenesis in the ME. We present these findings in the context of other studies on
\end{abstract}

\footnotetext{
S. Blackshaw $(\varangle)$

Solomon H. Snyder Department of Neuroscience, Johns Hopkins University School of Medicine, Baltimore, MD, USA

Institute for Cell Engineering, Johns Hopkins University School of Medicine, Baltimore, MD, USA
}

Department of Ophthalmology, Johns Hopkins University School of Medicine, Baltimore, MD, USA

Department of Neurology, Johns Hopkins University School of Medicine, Baltimore, MD, USA

Center for Human Systems Biology, Johns Hopkins University School of Medicine, Baltimore, MD, USA

e-mail: sblack@jhmi.edu

D.A. Lee

Solomon H. Snyder Department of Neuroscience, Johns Hopkins University School of Medicine, Baltimore, MD, USA

Division of Biology and Biomedical Engineering, California Institute of Technology,

Pasadena, CA, USA

T. Pak $\bullet$ S. Yoo

Solomon H. Snyder Department of Neuroscience, Johns Hopkins University School of Medicine, Baltimore, MD, USA

D. Pfaff, Y. Christen (eds.), Stem Cells in Neuroendocrinology, Research and

Perspectives in Endocrine Interactions, DOI 10.1007/978-3-319-41603-8_5 
the cellular and molecular mechanisms that regulate neurogenesis in postnatal and adult hypothalamus.

\section{Introduction}

Obesity and metabolic disorders are severe public health problems in developed countries. The pathophysiological effects of metabolic disease are partially mediated by hypothalamic inflammation (Thaler et al. 2012; Cai 2013; Purkayastha and Cai 2013) and by compensatory changes in hypothalamic neural circuitry triggered by obesity-induced neural injury. Recent studies have revealed hypothalamic neural injury in obese patients (Thaler et al. 2012). An understanding of the cellular and molecular responses to hypothalamic injury induced by dietary factors may identify new therapeutic targets for treating obesity and metabolic disorders (Lee and Blackshaw 2012).

Newborn neurons in the postnatal and adult hypothalamus have been described in multiple vertebrate species, including zebrafish (Wang et al. 2012; McPherson et al. 2016), chick (Kisliouk et al. 2014), hamster (Mohr and Sisk 2013), mouse (Lee et al. 2012), rats (Matsuzaki et al. 2015), and sheep (Batailler et al. 2015). This finding suggests a degree of plasticity that is evolutionarily conserved and likely extends to humans as well (Batailler et al. 2013; Dahiya et al. 2011; Noguiera et al. 2014). Both juvenile and adult mammalian hypothalamus exhibit ongoing neurogenesis that can be regulated by growth and differentiation factors (Pencea et al. 2001; Xu et al. 2005; Kokoeva et al. 2005; Perez-Martin et al. 2010; Robins et al. 2013b), diet (Lee et al. 2012; Li et al. 2012; McNay et al. 2012; Gouaze et al. 2013; Bless et al. 2014), antidepressants (Sachs and Caron 2014), exercise (Matsuzaki et al. 2015), and hormones (Ahmed et al. 2008; Bless et al. 2014). Although these studies generally agree that levels of constitutive hypothalamic neurogenesis are much lower than those seen in well-characterized neurogenic zones in the adult brain, such as the subventricular zone of the lateral ventricles or the subgranular zone of the lateral hypothalamus (Lee et al. 2012), they often report differing effects of extrinsic factors on cell proliferation and neurogenesis in hypothalamus. In addition, these studies make opposing claims about levels of neurogenesis and proliferation in certain hypothalamic regions, and the cell(s) of origin for these adult-born neurons remain controversial (Lee and Blackshaw 2012).

For instance, using a combination of in vitro cell culture and in vivo genetic lineage analysis, studies have claimed that a population of Sox2-positive (Li et al. 2012) and/or NG2-positive progenitors in the mediobasal hypothalamic parenchyma (Robins et al. 2013b) acts as multipotent neural progenitors. Tanycytes of the hypothalamic ventricular zone have also been reported to act as neural progenitors (Xu et al. 2005; Lee et al. 2012; Li et al. 2012; Haan et al. 2013; Robins 
et al. 2013a), and it has been variously claimed that dorsal located alpha2 and ventral beta2 tanycytes of the median eminence show the greatest levels of neurogenic potential (Robins et al. 2013a; Lee et al. 2012).

Other studies have reported that a range of extrinsic factors, such as dietary and hormonal signals as well as growth and differentiation factors, can also modulate postnatal hypothalamic neurogenesis. High-fat diet (HFD) has been reported to constitutively inhibit neurogenesis in the mediobasal hypothalamic parenchyma (Li et al. 2012; McNay et al. 2012) while activating neurogenesis in median eminence (ME; Lee et al. 2012; Hourai and Miyata 2013). It has also been reported that neurogenesis occurs in a sexually dimorphic pattern during puberty in hypothalamic regions, such as the preoptic area and anterioventral paraventricular nucleus that control sexual behavior (Ahmed et al. 2008), although the source of these young adult-generated neurons was not investigated.

Although these results seem discrepant at first glance, a closer examination reveals that these observed effects may result from methodological differences among the studies (Lee et al. 2012; Migaud et al. 2010). For instance, while multiple groups have reported that long-term administration of HFD inhibits cell proliferation and neurogenesis in hypothalamic parenchyma (Li et al. 2012; McNay et al. 2012; Gouaze et al. 2013; Bless et al. 2014), studies investigating acute responses to HFD have reported increased hypothalamic cell proliferation and neurogenesis (Thaler et al. 2012; Gouaze et al. 2013). Acute HFD administration has also been reported to rapidly induce hypothalamic inflammation, increasing cytokine signaling (Thaler et al. 2012). The physiological response in acute versus chronic HFD administration may serve different, but equally important, roles in maintaining metabolic homeostasis.

Hypothalamic progenitor cell populations may likewise respond differentially, and in some cases with opposite reactions, to dietary signals such as HFD. The ME, for instance, lies outside the blood-brain barrier; it is thus exposed to higher effective concentrations of circulating dietary and hormonal cues than the hypothalamic parenchyma (Fry et al. 2007; Langlet et al. 2013b). In contrast, all tanycyte subtypes directly contact the cerebrospinal fluid (CSF) and can potentially respond to intracerebral ventricular signals (Bennett et al. 2009; Bolborea and Dale 2013).

The age of the mice used for these studies has ranged from early postnatal (Lee et al. 2012) to young adult (Lee et al. 2012; Ahmed et al. 2008), to 3-12 months of age (Lee et al. 2012; Kokoeva et al. 2005; McNay et al. 2012; Kokoeva et al. 2007). Finally, studies of postnatal and adult neurogenesis in the ventrobasal hypothalamus have typically used either only male (Kokoeva et al. 2005, 2007; McNay et al. 2012; Li et al. 2012; Sachs and Caron 2014) or only female (Lee et al. 2012; Bless et al. 2014) mice. Neurogenesis in other hypothalamic regions is sexually dimorphic (Ahmed et al. 2008), making this but one additional methodological difference that could contribute to differences in the levels, location and dietary regulation of hypothalamic neurogenesis reported in these studies.

To clarify the extent to which sex-dependent factors might regulate neurogenesis in different hypothalamic regions, we investigated levels of hypothalamic neurogenesis in both the arcuate nucleus ( $\mathrm{ArcN})$ and $\mathrm{ME}$ in male and female 
young adult mice fed normal chow and HFD. We also investigated the effects of low-protein diet (LPD) and caloric restriction (CR) in these same areas in female mice. These dietary treatments led to significant and region-specific differences in neurogenesis. Most notably, HFD treatment inhibited ArcN neurogenesis in both sexes while selectively stimulating ME neurogenesis in female mice. In mice fed HFD, we found that inhibiting ME neurogenesis by computer tomography-guided focal irradiation attenuated weight gain in females but not males. These findings advance our understanding of extrinsic factors that regulate adult hypothalamic neurogenesis and reconcile a number of seemingly discrepant recent studies on this topic.

\section{Material and Methods}

\section{Animals}

Five week old female or male C57BL/6 mice were obtained from Charles River and housed in a 14/10-h light-dark cycle with free access to normal chow (Teklad F6 Rodent Diet 8664:: Protein (kcal): $31 \%$, Carbohydrate (kcal): $50 \%$, Fat (kcal): $19 \%$, Harlan Teklad, Madison, WI) and water. Where indicated, animals were provided with a high-fat diet (HFD) (Catalog \#: D12492i:: Protein (kcal): 20\%, Carbohydrate (kcal): $20 \%$, Fat (kcal): $60 \%$, Research Diets, New Brunswick, NJ) or low protein diet (Catalog\#: D11112203:: Protein (kcal): 8\%, Carbohydrate (kcal): $76 \%$, Fat (kcal): $16 \%$, Research Diets, New Brunswick, NJ). All mice used in these studies were maintained and euthanized according to protocols approved by the Institutional Animal Care and Use Committee at the Johns Hopkins School of Medicine.

\section{Caloric Restriction}

Five-week-old female C57BL/6 mice were obtained from Charles River and put on a high-fat diet (HFD: $60 \%$ kcals from fat, Research Diets \#D12492). At six weeks old, mice were separated into two groups: (control group) HFD ad libitum and HFD caloric restriction (CR). CR is at $70 \%$ of the HFD control group's average food intake. This was calculated by providing the amount of the control group's average food intake, plus an amount equal to the standard error of that group's intake, to ensure that mice would have enough food both to eat, and to spill, and maintain $70 \%$ of the HFD control group's average food intake. Food intakes were measured twice per week and used to calculate the CR levels to be used for 0.5 weeks until the next food intake assessment. 


\section{Reagents}

\section{Bromodeoxyuridine (BrdU)}

Where indicated, young adult mice received bromodeoxyuridine (BrdU; Sigma) administrated in the morning and evening by intraperitoneal injection at $50 \mathrm{mg} / \mathrm{kg}$ of body weight from P45 to P53.

\section{Tissue Processing and Antibodies}

Adult mice were sacrificed, perfused with $4 \%$ PFA/PBS, and cryoprotected as previously described (Lee et al. 2012). Serial sections (40 $\mu \mathrm{m}$ thick) were collected and stored at $-20^{\circ} \mathrm{C}$. Free-floating sections were immunostained using the following primary antibodies and working concentrations: mouse monoclonal anti-phospho-H2AX, Ser139, clone JBW301 (1:700, Millipore), rat monoclonal anti-BrdU (1:200, Accurate, Westbury, NY), mouse monoclonal anti-Hu $(5 \mu \mathrm{g} / \mathrm{ml}$, Molecular Probes, Carlsbad, CA). Double staining was visualized with Alexa Fluor 555-, and Alexa Fluor 488 (1:500, Molecular Probe, Carlsbad, CA). 4',6-diamidino2-phenylindole (DAPI) was used as a nuclear counterstain.

\section{Immunohistochemistry}

$\gamma \mathrm{H} 2 \mathrm{AX}$ immunostaining was performed as previously described (Lee et al. 2013). For BrdU immunostaining, sections were first incubated in $2 \mathrm{~N} \mathrm{HCl}$ at $37{ }^{\circ} \mathrm{C}$ for $30 \mathrm{~min}$, and rinsed in $0.1 \mathrm{M}$ boric acid $(\mathrm{pH} 8.5)$ at room temperature for $10 \mathrm{~min}$. Sections were then rinsed in PBST, blocked for $5 \mathrm{~min}$ in SuperBlock (ScyTek), and incubated overnight with in anti-BrdU antibody in $5 \%$ normal horse serum in PBS $/ 0.16 \%$ Triton X-100 at $4{ }^{\circ} \mathrm{C}$ in blocking solution. Sections were washed in PBST, incubated with secondary antibodies in blocking solution at RT for $2 \mathrm{~h}$, washed in PBST, mounted on Superfrost Plus slides (Fisher, Hampton, NH), and coverslipped with Gelvatol mounting medium.

\section{Cell Quantification}

All tissue sections used for quantification were imaged using confocal microscopy (Meta 510, Zeiss Microscopy). ME cells were counted. The dorsal-ventral boundary of the cells counted was the third ventricle $(3 \mathrm{~V})$ floor and the ventral edge of the external layer of the ME. The lateral boundaries were a $20 \mu \mathrm{m}$ medial inset off the corner of the $3 \mathrm{~V}$. ME dorsal and ventral boundaries remained identical to as previously described. Seven $40-\mu \mathrm{m}$ coronal serial sections $(280 \mu \mathrm{m})$ were counted 
between $-1.515 \mathrm{~mm}$ and $-1.875 \mathrm{~mm}$ from Bregma. On the rare occasion, a section would be lost in the collection process. If available, the next section in the mouse sample was taken and counted (seven sections were counted total). For analysis of newborn $\mathrm{Hu}^{+}$neurons, for each section analyzed, $\mathrm{Hu}^{+} \mathrm{DAPI}^{+}$and $\mathrm{Hu}^{+} \mathrm{BrdU}^{+} \mathrm{DAPI}{ }^{+}$ neurons within the ME were counted in the region defined above, excluding cells of the uppermost focal plane to avoid oversampling. To determine the frequency of $\mathrm{BrdU}^{+}$cells expressing $\mathrm{Hu}$, dual fluorescence-labeled sections were examined by confocal microscopy using a $20 \times$ objective and $1.5 \times$ digital zoom. For each marker and treatment condition, seven representative serial sections from each animal were examined. Sections were scored for double labeling by manual examination of optical slices. Cells were considered positive for a given phenotypic marker when the marker-specific labeling was unambiguously associated with a $\mathrm{BrdU}^{+}$nucleus. Cells were spot-checked in all three dimensions by Z-stack using a 63x objective. Images of $\mathrm{Hu}^{+} \mathrm{BrdU}^{+} \mathrm{DAPI}^{+}$labeling in feeding conditions (Fig. 1b) were blinded prior to counting. Cell counts are described in the text and figure legends as mean of several samples \pm s.e.m., total cell counts, and the number of samples examined to derive those total cell counts.

\section{Focal Irradiation of Ventrobasal Hypothalamus}

Radiation (10 Gy) was delivered using the Small Animal Radiation Research Platform (SARRP), a dedicated laboratory focal radiation device with CT capabilities (Xstrahl, Inc.). A detailed video and protocol describing this focal irradiation methodology is available online (http://www.jove.com/video/50716/functionalinterrogation-adult-hypothalamic-neurogenesis-with-focal) (Lee et al. 2013). Sham controls were performed in parallel. Mice in this cohort were caged, transported to the procedure room, received the same anesthesia, and received similar amounts of ambient radiation coming from the SARRP as the irradiated cohort. Sham controls differed only in that they did not receive a direct radiation beam and CT scan.

\section{Longitudinal Collection of Weight Data}

Weight data from each mouse subject was collected at the time of sham or irradiation treatment. Longitudinal weight gain was normalized to weight at the time of treatment for each subject. Weight data was taken every 0.5 weeks for each mouse subject. Female mice were group housed. Male mice were all group-housed initially, but were separated if they were observed to fight. To reduce variation resulting from changes in housing, the numbers of male mice that were individually and group housed were matched at all times between the sham and irradiated cohorts. For one experiment, HFD-fed female sham or irradiated cohorts, blood 
A

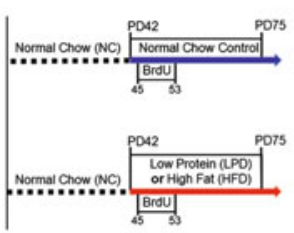

B

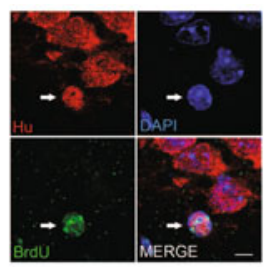

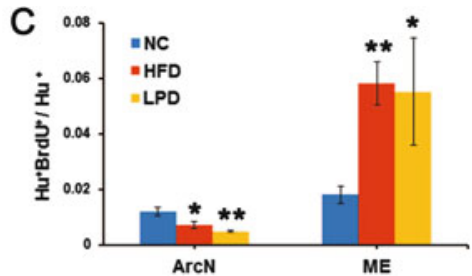

D
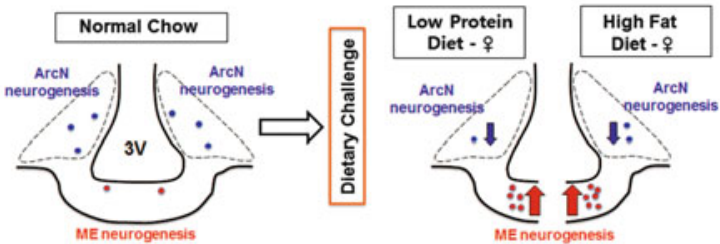

E
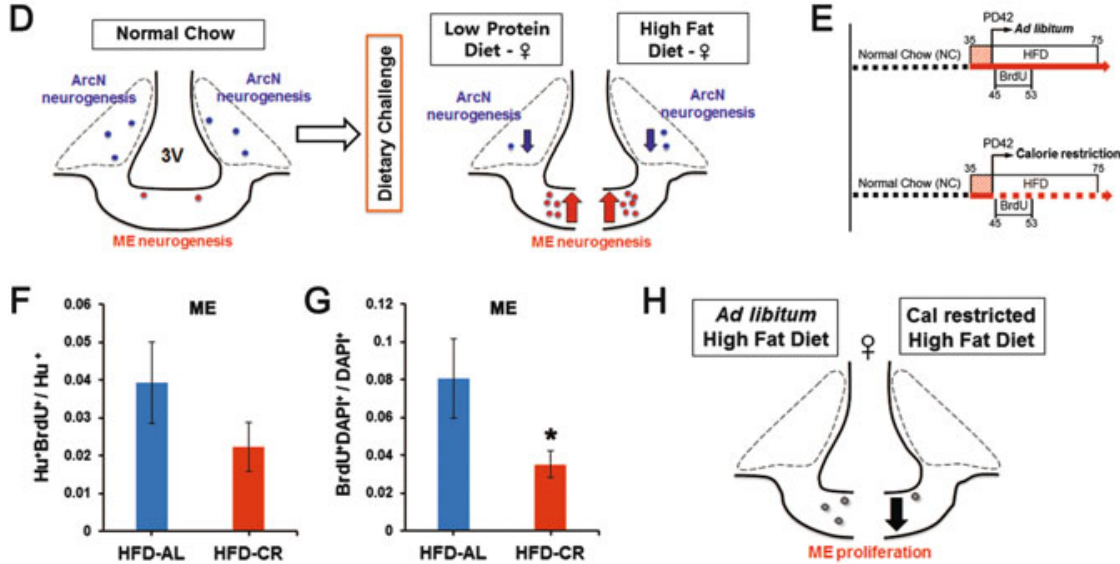

Fig. 1 Dynamic alteration of hypothalamic neurogenesis in response to different dietary conditions. (a) Experimental design schematic. Female mice were either continuously fed on normal chow (NC) or switched to the low protein diet (LPD) or high fat diet (HFD) at postnatal day (PD) 42. After 3 days, BrdU was injected intraperitoneally twice per day for 9 days. Mice were sacrificed 1 month after the onset of BrdU administration. (b) Representative high magnification image of BrdU and Hu double-positive P75 adult-born neurons (white arrows) located in median eminence (ME) of female mice fed HFD. (c) Quantitative comparison of diet-dependent neurogenesis $\left(\mathrm{Hu}^{+} \mathrm{BrdU}^{+} / \mathrm{Hu}^{+}\right.$neurons) in the arcuate nucleus $(\mathrm{ArcN})$ and $\mathrm{ME}$. (d) Schematic summarizing opposite effects of dietary change on neurogenesis between $\operatorname{ArcN}$ and ME. (e) Scheme of experimental design for calorie restriction. After 1 week initial adjustment to HFD (red striped square), female mice were fed either ad libitumHFD or were calorie restricted (CR) on the HFD to $70 \%$ of the ad libitum-fed mice, from PD42 onward. After 3 days, BrdU was injected intraperitoneally twice per day for 9 days. Mice were sacrificed 1 month after BrdU administration onset. (f, $\mathbf{g}$ ) Quantitative comparison of $\mathrm{ME}$ neurogenesis $\left(\mathrm{Hu}^{+} \mathrm{BrdU}^{+} / \mathrm{Hu}^{+}\right.$neurons) or proliferation $\left(\mathrm{BrdU}^{+} \mathrm{DAPI}^{+} / \mathrm{DAPI}^{+}\right.$cells) in CR HFD-fed (HFD-CR) mice or ad libitum HFD-fed (HFD-AL) mice. (h) Schematic summarizing significant reduction of cell proliferation in the ME of mice fed on HFD-CR. ${ }^{*} \mathrm{P}<0.05, * * \mathrm{P}<0.003$. Scale bar: $5 \mu \mathrm{m}$

samples were collected 1 week following treatment and a standard complete blood count panel was taken. There was no statistically significant difference in any of blood components. 


\section{Statistical Analysis}

Figures are shown as mean \pm standard error of the mean. Two-tailed Student's $t$-test was applied. A p-value $\leq 0.05$ indicated significant group difference.

\section{Results}

\section{Dietary Signals Differentially Regulate Neurogenesis and Cell Proliferation in ME and ArcN}

Our group previously demonstrated that feeding young adult female mice HFD led to significantly increased ME neurogenesis (Lee et al. 2012). We set out to test whether additional dietary conditions could also alter neurogenesis in the ME and whether these conditions led to comparable changes in neurogenesis in the ArcN, which lies inside the blood-brain barrier within the hypothalamus proper. Young adult female mice were switched onto control normal chow (NC), HFD or LPD beginning at postnatal day (P) 42. Cell proliferation was tracked using twice-daily intraperitoneal (i.p.) injections of BrdU from P45-53 (Fig. 1a). Mice were euthanized at P75, and brains were immunostained for $\mathrm{BrdU}$ and the pan-neuronal marker $\mathrm{HuC} / \mathrm{D}$. The fraction of $\mathrm{Hu}^{+}$cells that were also $\mathrm{BrdU}^{+}$was quantified to assess levels of neurogenesis.

Baseline levels of hypothalamic neurogenesis $\left[\left(\mathrm{Hu}^{+} \mathrm{BrdU}^{+}\right) / \mathrm{Hu}^{+}\right.$neurons $]$in mice fed $\mathrm{NC}$ were low and did not differ significantly between the two regions $(\mathrm{NC} \operatorname{ArcN}[0.012 \pm 0.002, \mathrm{n}=5]$ vs NC ME $[0.015 \pm 0.004, \mathrm{n}=7], \mathrm{p}=0.47$, equal variance; Fig. 1c). Both HFD-fed mice $(0.0072 \pm 0.0012, \mathrm{n}=5, \mathrm{p}=0.044$, equal " $n$ ") and LPD-fed mice ( $0.0048 \pm 0.0004, \mathrm{n}=5, \mathrm{p}=0.0022$, equal " $\mathrm{n}$ ") showed a substantial reduction in the fraction of $\mathrm{Hu}^{+} \mathrm{BrdU}^{+} \mathrm{ArcN}$ neurons. In contrast, both HFD-fed mice $(0.058 \pm 0.008, \mathrm{n}=9, \mathrm{p}=0.0005$, unequal " $\mathrm{n}$," equal variance) and LPD-fed mice $(0.055 \pm 0.019, \mathrm{n}=4, \mathrm{p}=0.025$, unequal " $\mathrm{n}$," equal variance) showed a significant increase in the fraction of $\mathrm{Hu}^{+} \mathrm{BrdU}^{+} \mathrm{ME}$ neurons (Fig. 1c). The differences in neurogenesis levels between the $\mathrm{ArcN}$ and $\mathrm{ME}$ following both $\mathrm{HFD}(\operatorname{ArcN}[0.0072 \pm 0.001, \mathrm{n}=5]$ vs. ME [0.058 $\pm 0.008, \mathrm{n}=9], \mathrm{p}=0.0005$, equal variance) and $\mathrm{LPD}(\mathrm{ArcN}[0.0048 \pm 0.0004, \mathrm{n}=5]$ vs. $\mathrm{ME}[0.055 \pm .019$, $\mathrm{n}=4], \mathrm{p}=0.021$, equal variance] were significant and implied that neural progenitor populations in these two regions responded differentially to these dietary cues (Fig. 1c, d). The HFD-induced inhibition of ArcN neurogenesis was similar to observations of adult male mice made by other groups (McNay et al. 2012; Li et al. 2012).

Because previous studies reported that CR could reverse the effects of HFD on ArcN neurogenesis (McNay et al. 2012), we next tested whether CR could likewise modulate HFD-induced ME neurogenesis. For these studies, female mice were housed individually and allowed either ad libitum or restricted access to HFD 
starting at P45. Restricted HFD access was at $70 \%$ of the caloric intake of animals fed ad libitum (Fig. 1e). BrdU labeling and immunohistochemistry were conducted as described above. CR HFD-fed mice trended towards a decrease in neurogenesis levels in ME (HFD ad lib [0.039 $\pm 0.011, \mathrm{n}=4]$ vs HFD-CR $[0.022 \pm 0.006, \mathrm{n}=6]$ $\mathrm{p}=0.19$, equal variance), but this effect did not reach significance (Fig. 1f). However, we observed that overall $\mathrm{BrdU}$ incorporation in ME cells was significantly reduced (HFD ad lib $[0.08 \pm 0.02, \mathrm{n}=4]$ vs HFD-CR $[0.035 \pm 0.007, \mathrm{n}=6]$, $\mathrm{p}=.043$, equal variance) (Fig. $1 \mathrm{~g}$ ). We thus conclude that CR inhibits proliferation of progenitor cells within the ME (Fig. 1h).

\section{Sex-Specific Differences in Diet-Induced Hypothalamic Neurogenesis}

Since these studies were all performed in young adult females, we next tested whether the levels of baseline and HFD-induced ME neurogenesis were different between the sexes. We used diet and BrdU labeling conditions identical to those in Fig. 1a to measure levels of neurogenesis in male mice fed either NC or HFD. We observed that young adult males fed NC showed levels of neurogenesis in both ArcN and ME that were low and not significantly different from age-matched females (NC-fed: Male $\operatorname{ArcN}[0.0119 \pm 0.0012, n=3]$ vs Male ME $[0.020 \pm 0.009, n=3], p=0.40$, equal variance; Fig. 2a). We likewise observed that male mice fed HFD showed reduced levels of $\mathrm{ArcN}$ neurogenesis relative to male mice fed $\mathrm{NC}$ ( NC ArcN $[0.0119 \pm 0.0016, \mathrm{n}=3]$ vs HFD ArcN $[0.0070 \pm 0.0011, \mathrm{n}=5] \mathrm{p}=0.036$, equal variance). However, in sharp contrast to what we observed in females, male mice showed no increase in ME neurogenesis when fed HFD $(0.020 \pm 0.009, \mathrm{n}=3$, vs $0.025 \pm 0.006, \mathrm{n}=4: \mathrm{NC}$ vs HFD, $\mathrm{p}=0.36$; Fig. 2a), implying that neural progenitor populations in the ME showed sex-specific increases in neurogenesis in response to HFD (Fig. 2b).
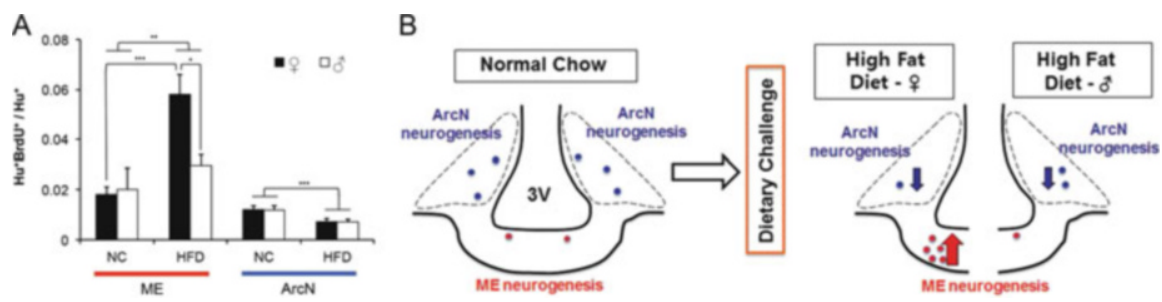

Fig. 2 Sex differences in neurogenic response to HFD. (a) Quantification of hypothalamic neurogenesis $\left(\mathrm{Hu}^{+} \mathrm{BrdU}^{+} / \mathrm{Hu}^{+}\right.$neurons) in the $\mathrm{ArcN}$ and $\mathrm{ME}$ of young adult male mice fed HFD or NC. (b) Schematic summarizing sexual dimorphism of dietary challenge on ME neurogenesis 


\section{Blocking Neurogenesis in ME Attenuates HFD-Induced Weight Gain in Young Adult Female, But Not Male, Mice}

We previously demonstrated that computer tomography-guided focal irradiation could selectively inhibit cell proliferation in the ME while sparing proliferation in ArcN (Lee et al. 2012, 2013). Our radiological approach reduced ME neurogenesis by $\sim 85 \%$ (Lee et al. 2012), in line with previous approaches that used focal irradiation in other mammalian neurogenic niches (Ford et al. 2011). We next tested whether selective radiological inhibition of ME neurogenesis (Fig. 3a) in both males and female mice fed HFD led to sex-specific differences in regulation of body weight. The specificity of the focal irradiation was demonstrated using $\gamma$ H2AX immunostaining (Fig. 3b; Lee et al. 2012, 2013). Longitudinal body weight measurements were then taken for male and female mice that underwent either focal irradiation or sham treatment and that were fed either NC or HFD. Weight changes were normalized to the starting weight of each mouse at the time of sham or irradiation treatment.
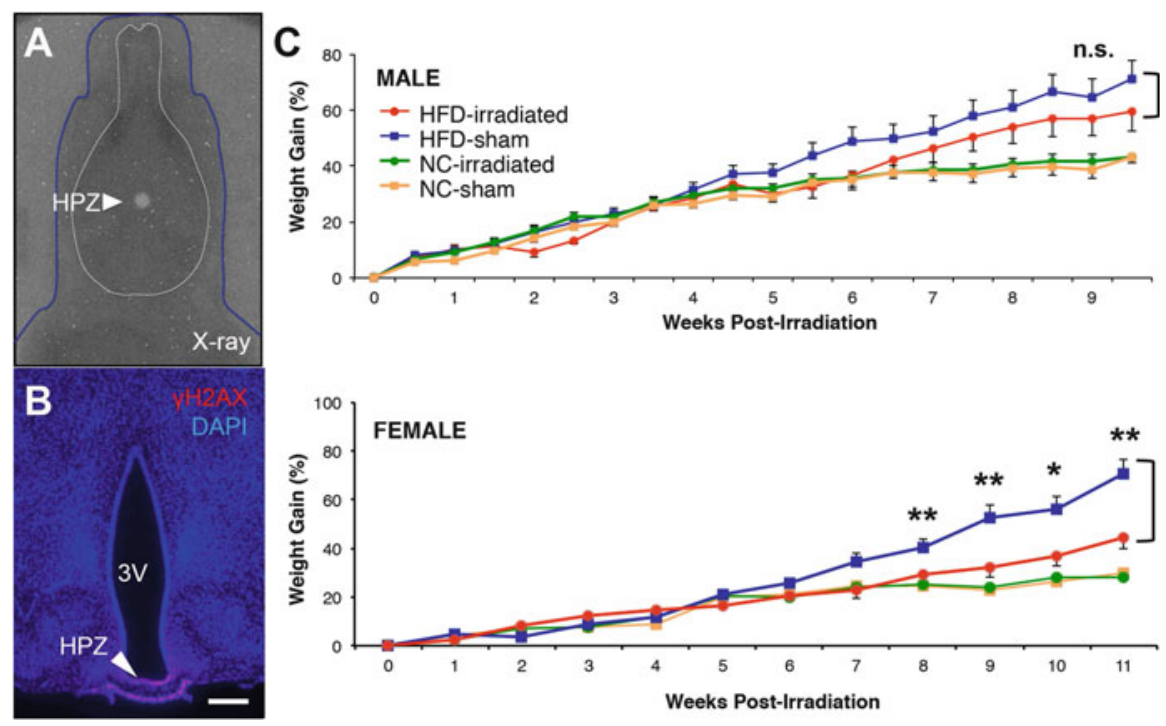

Fig. 3 Sex differences in diet-induced weight gain after focal inhibition of ME neurogenesis. Mice received either NC or HFD at 5 weeks of age. Sham treatment or 10 Gy of computer tomography-guided focal radiation was applied to the ME of young adult mice (P42) as previously described (Lee et al. 2013). (a) Superimposition of dosimetry-film acquired with 1-mm radiation beam in phantom with an X-ray of a real mouse subject (blue line). White circle (arrow) indicates 10-Gy radiation dose focally targeted to hypothalamic proliferative zone (HPZ). (b) Confirmation of radiation targeting accuracy by $\gamma \mathrm{H} 2 \mathrm{AX}$ immunostaining, an indicator of DNA double strand breaks and radiation localization. (c) Weight gain was assessed from the time of either sham or focal irradiation treatment in NC- and HFD-fed mice of both sexes 
We observed no long-term changes in body weight between sham and irradiated animals fed NC in either our male (sham treatment: $n=8$; irradiation treatment: $\mathrm{n}=12$ ) or female (sham treatment: $\mathrm{n}=12$; irradiation treatment: $\mathrm{n}=12$ ) cohorts (Fig. 3c). In contrast, HFD-fed female mice showed a significant reduction in weight gain following irradiation relative to sham controls, as previously reported (Lee et al. 2012). At 9 weeks post-treatment, irradiated female mice receiving HFD $(n=10)$ had a $32 \pm 4 \%$ increase in weight gain relative to sham controls $(n=9)$, which showed a $52 \pm 6 \%$ increase in weight gain (p-value $=0.028$, Fig. $3 \mathrm{c}$ ). In sharp contrast, no significant differences in weight gain were observed in irradiated HFD-fed males relative to sham controls ( $59 \pm 7 \%$ increase, $\mathrm{n}=11$, vs $71 \pm 7 \%$ increase, $n=12, p=0.27$; Fig. $3 \mathrm{c}$ ). These data confirm previous reports that neurons generated in the female ME in response to HFD act to promote energy storage (Lee et al. 2012, 2013).

\section{Discussion}

Several recent studies have reported that neurogenesis occurs in the adult hypothalamus (Migaud et al. 2010; Lee and Blackshaw 2012, 2014), a central regulator of metabolism and energy balance. We investigated how changes in diet can modulate hypothalamic neurogenesis by presenting young adult mice with different diets. We observed that HFD, LPD, and CR HFD all differentially modulated proliferation and neurogenesis in the hypothalamic $\mathrm{ME}$ and $\mathrm{ArcN}$, hypothalamic regions that regulate energy balance (summarized in Fig. 4b). We observed an increase in $\mathrm{ME}$ neurogenesis and a decrease in $\mathrm{ArcN}$ neurogenesis in response to both HFD and LPD (Fig. 1c, d). In contrast, HFD selectively activated neurogenesis in the female ME. These differential region-specific changes in neurogenesis may lead to differential generation of orexinergic and anorexinergic neurons in response to dietary cues and may serve as a mechanism that allows adaptation to long-term changes in energy balance homeostasis.

These region-specific changes in adult hypothalamic neurogenesis most likely are mediated by differing exposures to secreted peptide, growth factors, and neurotrophic factors that signify feeding status and long-term energy availability. The ME, by virtue of its access to the third ventricle and status as a circumventricular organ, is exposed to a variety of these secreted factors via the CSF and the blood. By comparison, the $\operatorname{ArcN}$, a structure protected by the bloodbrain barrier (Mullier et al. 2010), has less access to circulating satiety signals and hormones. The permeability of hypothalamic blood-brain barrier to blood-borne factors has been proposed to be differentially regulated in fed and fasting conditions through a VEGF-dependent mechanism (Langlet et al. 2013a). Continuous integration of these peripheral signals by neurons belonging to both the $\operatorname{ArcN}$ and the $\mathrm{ME}$ of the hypothalamus is critical for central regulation of energy balance and neuroendocrine function (Schaeffer et al. 2013). Our data suggest that adult-generated 


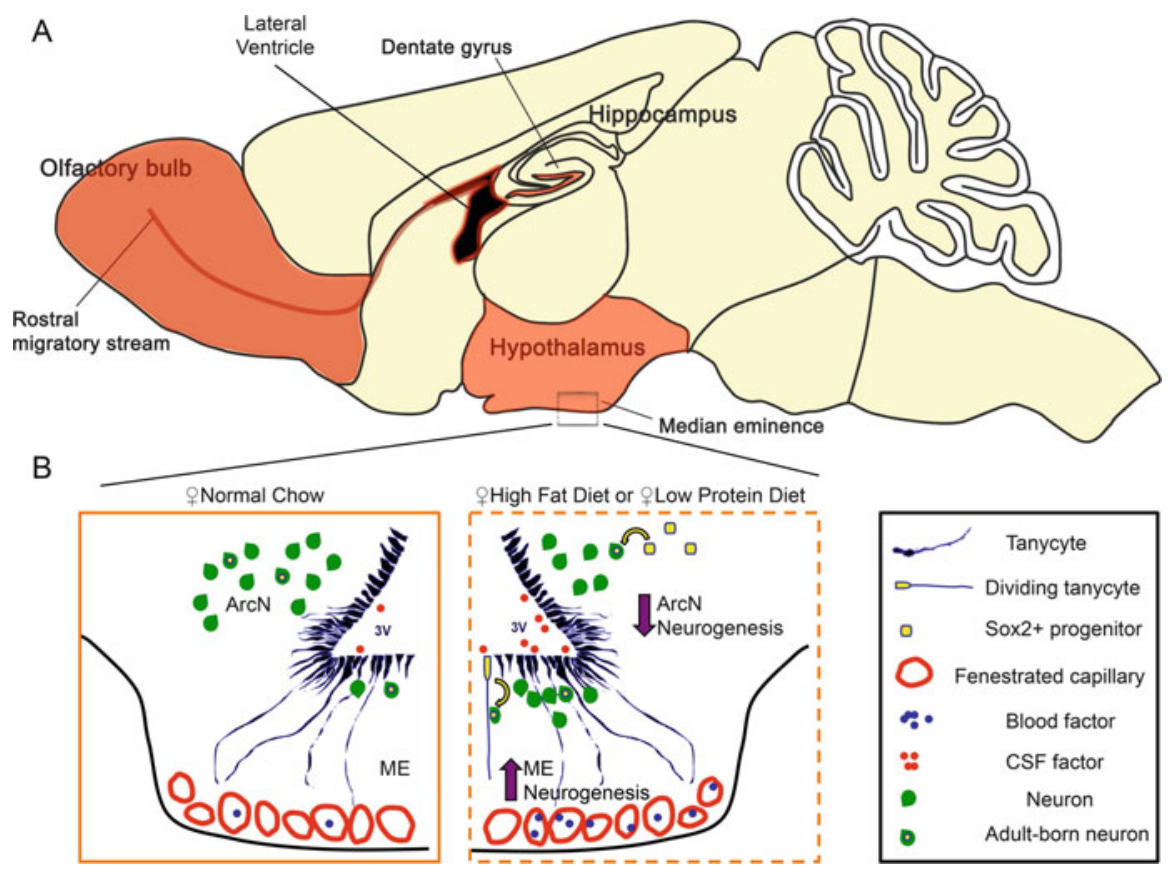

Fig. 4 Regulation of hypothalamic neurogenesis by dietary factors. (a) In mammals, constitutive adult neurogenesis is primarily confined to three brain regions (highlighted in orange). The hippocampal dentate gyrus and the subventricular zone of the lateral ventricles are canonical neurogenic niches. Additionally, recent observations demonstrate that the ventrobasal hypothalamus serves as a neurogenic niche, engaging in low but constitutive levels of neurogenesis in adults. (b) Hypothalamic tanycytes and parenchymal Sox $2^{+}$cells represent potential hypothalamic neural progenitor populations that give rise to adult-born neurons. In the hypothalamic ME, tanycytes are a convincing neurogenic source for the ArcN and ME. In females, both HFD and low protein diet (LPD) increase ME neurogenesis in the ME, while concurrently decreasing ArcN neurogenesis in the ArcN. Several lines of evidence suggest that this dynamic change in neurogenesis is mediated between a tanycytic neural progenitor pool and factors present in the cerebrospinal fluid (CSF) and/or those circulating through the fenestrated capillaries of the ME

neurons in both hypothalamic regions may show differing sensitivities to dietary and hormonal signals that help maintain energy homeostasis.

Several secreted factors that signal feeding status and long-term energy availability regulate adult neurogenesis in various neurogenic niches, including the hypothalamus (Sousa-Ferreira et al. 2014). For instance, compared to NC-fed controls, HFD-fed mice exhibited substantially higher ciliary neurotrophic factor (CNTF) mRNA in tanycytes and multi-ciliated ependymal cells, whereas CR mice showed substantially lower expression levels; this coincided with similar changes in CNTF receptor (CNTFR) mRNA (Severi et al. 2013). Taken together with previous findings that CNTF delivered by i.c.v. cannulation stimulates adult hypothalamic neurogenesis (Kokoeva et al. 2005), this finding suggests that dietary signals may regulate hypothalamic neurogenesis in the ME through altered CNTF 
signaling. This hypotheses is supported by observations that beta2 tanycytes of the adult hypothalamic proliferative zone (HPZ) are enriched with CNTFR, as compared to alpha 1,2 tanycytes (Kokoeva et al. 2005). In that study, mice receiving intracerebroventricular infusion of CNTF demonstrated hyperplasia within the HPZ of the ME, as indicated by particularly high levels of BrdU incorporation (Kokoeva et al. 2005). The development of inducible Cre mouse lines specific for hypothalamic neural progenitors will help identify additional signaling pathways that are critical for the regulation of hypothalamic neurogenesis (Robins et al. 2013a, b; Pak et al. 2014).

In female mice fed a NC diet, we observed relatively low levels of hypothalamic neurogenesis in the ME. Interestingly, upon presentation of a dietary challenge such as HFD, ME neurogenesis was increased in females. Moreover, computer tomography-guided radiological inhibition of ME neurogenesis (Lee et al. 2013) reduced HFD-induced weight gain in females, but not in males (Fig. 3b). These intriguing results suggest that weight gain in females can be attributed in part to additional adult-generated neurons in the $\mathrm{ME}$ and that the neural circuitry regulating body weight differs in some respects between females and males. These findings are consistent with previous studies demonstrating that sex hormones can regulate hypothalamic neurogenesis in a region-dependent manner (Ahmed et al. 2008; Cheng 2013). Taken together with our results, this body of work highlights the importance of examining both the regional differences in hypothalamic neurogenesis and the sex-specific differences. Such differences likely at least partially account for differences in the levels and diet dependence of adult hypothalamic neurogenesis observed by different groups.

Factors that mediate these sex-dependent differences in hypothalamic neurogenesis have not yet been identified but could involve numerous levels of regulation, such as hormone-dependent plasticity (de Seranno et al. 2010), differences in blood-barrier access between the sexes (Hoxha et al. 2013), and hormonespecific induction of feeding behavior (Sieck et al. 1978). Lastly, it is possible that it is the survival of newborn neurons, rather than (or in addition to) their proliferation, that is sexually dimorphic, as has been previously demonstrated for prenatally generated hypothalamic neurons (Waters and Simerly 2009; Forger et al. 2004; Tobet and Hanna 1997; Park et al. 1998).

In addition to being a means of regulating energy homeostasis in response to long-term changes in diet, adult hypothalamic neurogenesis may be triggered in response to environmental injury. The hypothalamic ME, in contrast to other hypothalamic regions, lies outside of the blood-brain barrier and is thus directly exposed to circulating toxins and pathogens, as well as nutrients that can lead to cellular injury when in oversupply. Hypothalamic neural injury and inflammation are seen in obese animals and humans (Thaler et al. 2012, 2013; Li et al. 2012). The increased neurogenesis in adult female ME may serve to replace damaged neurons in this region. Indeed, at least one study has reported that neurons important for energy balance regulation can be regenerated in adult hypothalamus in response to neurodegenerative-like injury (Pierce and Xu 2010). Further studies to determine the role of environmental and physiological factors in regulating adult 
hypothalamic neurogenesis may yet reveal new mechanistic approaches towards the treatment of obesity and metabolic disorders.

Acknowledgments We would like to thank W. Yap, B. Clark and J. Bedont for their insightful comments on the manuscript. This work was supported by NIH R01DK108230 and a Baltimore Diabetes Research Center Training and Feasibility grant to S.B. S.B. was a W.M. Keck Distinguished Young Scholar in Medical Research.

Open Access This chapter is distributed under the terms of the Creative Commons Attribution 4.0 International License (http://creativecommons.org/licenses/by/4.0/), which permits use, duplication, adaptation, distribution and reproduction in any medium or format, as long as you give appropriate credit to the original author(s) and the source, a link is provided to the Creative Commons license and any changes made are indicated.

The images or other third party material in this chapter are included in the work's Creative Commons license, unless indicated otherwise in the credit line; if such material is not included in the work's Creative Commons license and the respective action is not permitted by statutory regulation, users will need to obtain permission from the license holder to duplicate, adapt or reproduce the material.

\section{References}

Ahmed EI, Zehr JL, Schulz KM, Lorenz BH, DonCarlos LL, Sisk CL (2008) Pubertal hormones modulate the addition of new cells to sexually dimorphic brain regions. Nat Neurosci 11:995-997

Batailler M, Droguerre M, Baroncini M, Fontaine C, Prevot V, Migaud M (2013) DCX expressing cells in the vicinity of the hypothalamic neurogenic niche: a comparative study between mouse, sheep and human tissues. J Comp Neurol 522:1966-1985

Batailler M, Derouet L, Butruille L, Migaud M (2015) Sensitivity to the photoperiod and potential migratory features of neuroblasts in the adult sheep hypothalamus. Brain Struct Funct [Epub ahead of print]

Bennett L, Yang M, Enikolopov G, Iacovitti L (2009) Circumventricular organs: a novel site of neural stem cells in the adult brain. Mol Cell Neurosci 41:337-347

Bless EP, Reddy T, Acharya KD, Beltz BS, Tetel MJ (2014) Oestradiol and diet modulate energy homeostasis and hypothalamic neurogenesis in the adult female mouse. J Neuroendocrinol 26:805-816

Bolborea M, Dale N (2013) Hypothalamic tanycytes: potential roles in the control of feeding and energy balance. Trends Neurosci 36:91-100

Cai D (2013) Neuroinflammation and neurodegeneration in overnutrition-induced diseases. Trends Endocrinol Metab 24:40-47

Cheng MF (2013) Hypothalamic neurogenesis in the adult brain. Front Neuroendocrinol 34:167-178

Dahiya S, da Lee Y, Gutmann DH (2011) Comparative characterization of the human and mouse third ventricle germinal zones. J Neuropathol Exp Neurol 70:622-633

de Seranno S, d'Anglemont de Tassigny X, Estrella C, Loyens A, Kasparov S, Leroy D, Ojeda SR, Beauvillain JC, Prevot V (2010) Role of estradiol in the dynamic control of tanycyte plasticity mediated by vascular endothelial cells in the median eminence. Endocrinology 151:1760-1772

Ford EC, Achanta P, Purger D, Armour M, Reyes J, Fong J, Kleinberg L, Redmond K, Wong J, Jang MH, Jun H, Song HJ, Quinones-Hinojosa A (2011) Localized CT-guided irradiation inhibits neurogenesis in specific regions of the adult mouse brain. Radiat Res 175:774-783 
Forger NG, Rosen GJ, Waters EM, Jacob D, Simerly RB, de Vries GJ (2004) Deletion of Bax eliminates sex differences in the mouse forebrain. Proc Natl Acad Sci U S A 101:13666-13671

Fry M, Hoyda TD, Ferguson AV (2007) Making sense of it: roles of the sensory circumventricular organs in feeding and regulation of energy homeostasis. Exp Biol Med (Maywood) 232:14-26

Gouaze A, Brenachot X, Rigault C, Krezymon A, Rauch C, Nedelec E, Lemoine GJ, Bauer S, Pénicaud L, Benani A (2013) Cerebral cell renewal in adult mice controls the onset of obesity. PLoS One 8, e72029

Haan N, Goodman T, Najdi-Samiei A, Stratford CM, Rice R, El Agha E, Bellusci S, Hajihosseini MK (2013) Fgf10-expressing tanycytes add new neurons to the appetite/energy-balance regulating centers of the postnatal and adult hypothalamus. J Neurosci 33:6170-6180

Hourai A, Miyata S (2013) Neurogenesis in the circumventricular organs of adult mouse brains. J Neurosci Res 91:757-770

Hoxha V, Lama C, Chang PL, Saurabh S, Patel N, Olate N, Dauwalder B (2013) Sex-specific signaling in the blood-brain barrier is required for male courtship in Drosophila. PLoS Genet 9, e1003217

Kisliouk T, Cramer T, Meiri N (2014) Heat stress attenuates new cell generation in the hypothalamus: a role for miR-138. Neuroscience 277:624-636

Kokoeva MV, Yin H, Flier JS (2005) Neurogenesis in the hypothalamus of adult mice: potential role in energy balance. Science 310:679-683

Kokoeva MV, Yin H, Flier JS (2007) Evidence for constitutive neural cell proliferation in the adult murine hypothalamus. J Comp Neurol 505:209-220

Langlet F, Levin BE, Luquet S, Mazzone M, Messina A, Dunn-Meynell AA, Balland Lacombe A, Mazur D, Carmeliet P, Bouret SG, Prevot V, Dehouck B (2013a) Tanycytic VEGF-A boosts blood-hypothalamus barrier plasticity and access of metabolic signals to the arcuate nucleus in response to fasting. Cell Metab 17:607-617

Langlet F, Mullier A, Bouret SG, Prevot V, Dehouck B (2013b) Tanycyte-like cells form a bloodcerebrospinal fluid barrier in the circumventricular organs of the mouse brain. J Comp Neurol 521:3389-3405

Lee DA, Blackshaw S (2012) Functional implications of hypothalamic neurogenesis in the adult mammalian brain. Int J Dev Neurosci 30:615-621

Lee DA, Blackshaw S (2014) Feed your head: neurodevelopmental control of feeding and metabolism. Annu Rev Physiol 76:197-223

Lee DA, Bedont JL, Pak T, Wang H, Song J, Miranda-Angulo A, Takiar V, Charubhumi V, Balordi F, Takebayashi H, Aja S, Ford E, Fishell G, Blackshaw S (2012) Tanycytes of the hypothalamic median eminence form a diet-responsive neurogenic niche. Nat Neurosci 15:700-702

Lee DA, Salvatierra J, Velarde E, Wong J, Ford EC, Blackshaw S (2013) Functional interrogation of adult hypothalamic neurogenesis with focal radiological inhibition. J Vis Exp (81):e50716

Li J, Tang Y, Cai D (2012) IKKbeta/NF-kappaB disrupts adult hypothalamic neural stem cells to mediate a neurodegenerative mechanism of dietary obesity and pre-diabetes. Nat Cell Biol 14:999-1012

Matsuzaki K, Katakura M, Inoue T, Hara T, Hashimoto M, Shido O (2015) Aging attenuates acquired heat tolerance and hypothalamic neurogenesis in rats. J Comp Neurol 523:1190-1201

McNay DE, Briancon N, Kokoeva MV, Maratos-Flier E, Flier JS (2012) Remodeling of the arcuate nucleus energy-balance circuit is inhibited in obese mice. J Clin Invest 122:142-152

McPherson AD, Barrios JP, Luks-Morgan SJ, Manfredi JP, Bonkowsky JL, Douglass AD, Dorsky RI (2016) Motor behavior mediated by continuously generated dopaminergic neurons in the zebrafish hypothalamus recovers after cell ablation. Curr Biol 26:263-269

Migaud M, Batailler M, Segura S, Duittoz A, Franceschini I, Pillon D (2010) Emerging new sites for adult neurogenesis in the mammalian brain: a comparative study between the hypothalamus and the classical neurogenic zones. Eur J Neurosci 32:2042-2052 
Mohr MA, Sisk CL (2013) Pubertally born neurons and glia are functionally integrated into limbic and hypothalamic circuits of the male Syrian hamster. Proc Natl Acad Sci U S A 110:4792-4797

Mullier A, Bouret SG, Prevot V, Dehouck B (2010) Differential distribution of tight junction proteins suggests a role for tanycytes in blood-hypothalamus barrier regulation in the adult mouse brain. J Comp Neurol 518:943-962

Nogueira AB, Sogayar MC, Colquhoun A, Siqueira SA, Nogueira AB, Marchiori PE, Teixeira MJ (2014) Existence of a potential neurogenic system in the adult human brain. J Transl Med 12:75

Pak T, Yoo S, Miranda-Angulo LA, Blackshaw S (2014) Rax-CreER ${ }^{\mathrm{T} 2}$ knock-in mice: a tool for selective and conditional gene deletion in progenitors and radial glia of the retina and hypothalamus. PLoS One 9, e90381

Park JJ, Tobet SA, Baum MJ (1998) Cell death in the sexually dimorphic dorsal preoptic area/ anterior hypothalamus of perinatal male and female ferrets. J Neurobiol 34:242-252

Pencea V, Bingaman KD, Wiegand SJ, Luskin MB (2001) Infusion of brain-derived neurotrophic factor into the lateral ventricle of the adult rat leads to new neurons in the parenchyma of the striatum, septum, thalamus, and hypothalamus. J Neurosci 21:6706-6717

Perez-Martin M, Cifuentes M, Grondona JM, Lopez-Avalos MD, Gomez-Pinedo U, GarciaVerdugo JM, Fernandez-Llebrez P (2010) IGF-I stimulates neurogenesis in the hypothalamus of adult rats. Eur J Neurosci 31:1533-1548

Pierce AA, Xu AW (2010) De novo neurogenesis in adult hypothalamus as a compensatory mechanism to regulate energy balance. J Neurosci 30:723-730

Purkayastha S, Cai D (2013) Neuroinflammatory basis of metabolic syndrome. Mol Metab 2:356-363

Robins SC, Trudel E, Rotondi O, Liu X, Djogo T, Kryzskaya D, Bourque CW, Kokoeva MV (2013a) Evidence for NG2-glia derived, adult-born functional neurons in the hypothalamus. PLoS One 8, e78236

Robins SC, Stewart I, McNay DE, Taylor V, Giachino C, Goetz M, Ninkovic J, Briancon N, Maratos-Flier E, Flier JS, Kokoeva MV, Placzek M (2013a) Alpha-Tanycytes of the adult hypothalamic third ventricle include distinct populations of FGF-responsive neural progenitors. Nat Commun 4: 2049

Sachs BD, Caron MG (2014) Chronic fluoxetine increases extra-hippocampal neurogenesis in adult mice. Int J Neuropsychopharmacol 18(4). doi:10.1093/ijnp/pyu029

Schaeffer M, Langlet F, Lafont C, Molino F, Hodson DJ, Roux T, Lamarque L, Verdié P, Bourrier E, Dehouck B, Banères JL, Martinez J, Méry PF, Marie J, Trinquet E, Fehrentz JA, Prévot V, Mollard P (2013) Rapid sensing of circulating ghrelin by hypothalamic appetitemodifying neurons. Proc Natl Acad Sci U S A 110:1512-1517

Severi I, Perugini J, Mondini E, Smorlesi A, Frontini A, Cinti S, Giordano A (2013) Opposite effects of a high-fat diet and calorie restriction on ciliary neurotrophic factor signaling in the mouse hypothalamus. Front Neurosci 7:263

Sieck GC, Nance DM, Gorski RA (1978) Estrogen modification of feeding behavior in the female rat: influence of metabolic state. Physiol Behav 21:893-897

Sousa-Ferreira L, de Almeida LP, Cavadas C (2014) Role of hypothalamic neurogenesis in feeding regulation. Trends Endocrinol Metab 25:80-88

Thaler JP, Yi CX, Schur EA, Guyenet SJ, Hwang BH, Dietrich MO, Zhao X, Sarruf DA, Izgur V, Maravilla KR, Nguyen HT, Fischer JD, Matsen ME, Wisse BE, Morton GJ, Horvath TL, Baskin DG, Tschöp MH, Schwartz MW (2012) Obesity is associated with hypothalamic injury in rodents and humans. J Clin Invest 122:153-162

Thaler JP, Guyenet SJ, Dorfman MD, Wisse BE, Schwartz MW (2013) Hypothalamic inflammation: marker or mechanism of obesity pathogenesis? Diabetes 62:2629-2634

Tobet SA, Hanna IK (1997) Ontogeny of sex differences in the mammalian hypothalamus and preoptic area. Cell Mol Neurobiol 17:565-601 
Wang X, Kopinke D, Lin J, McPherson AD, Duncan RN, Otsuna H, MoroE HK, Grunwald DJ, Argenton F, Chien CB, Murtaugh LC, Dorsky RI (2012) Wnt signaling regulates postembryonic hypothalamic progenitor differentiation. Dev Cell 23:624-636

Waters EM, Simerly RB (2009) Estrogen induces caspase-dependent cell death during hypothalamic development. J Neurosci 29:9714-9718

Xu Y, Tamamaki N, Noda T, Kimura K, Itokazu Y, Matsumoto N, Dezawa M, Ide C (2005) Neurogenesis in the ependymal layer of the adult rat 3rd ventricle. Exp Neurol 192:251-264 